\title{
A new insight into the nature of bonding in the dimers of Lappert's stannylene and its Ge analogs. A quantum mechanical study
}

\section{Supporting Information}

Robert Sedlak, ${ }^{[\mathrm{a}, \mathrm{b}]}$ Olga A. Stasyuk, ${ }^{[\mathrm{a}]}$ Célia Fonseca Guerra, ${ }^{[\mathrm{c}]}$ Jan Řezáč ${ }^{[\mathrm{a}]}$, Aleš Růžička*[d] and Pavel $\operatorname{Hobza}^{*[a, b]}$

\footnotetext{
${ }^{a}$ Institute of Organic Chemistry and Biochemistry, Academy of Sciences of the Czech Republic, 16610 Prague 6, Czech Republic ${ }^{\mathrm{b}}$ Regional Centre of Advanced Technologies and Materials, Department of Physical Chemistry, Palacký University, 77146 Olomouc, Czech Republic

${ }^{c}$ Department of Theoretical Chemistry and Amsterdam Center for Multiscale Modeling, VU Amsterdam, De Boelelaan 1083, 1081 HV Amsterdam, The Netherlands

${ }^{d}$ Department of General and Inorganic Chemistry, Faculty of Chemical Technology, University of Pardubice, Studentská 573, CZ532 10, Pardubice, Czech Republic
}

Figure S1. $\mathrm{MO}$ analyses for Sn-X dimers. Black numbers denotes $\mathrm{MO}$ energy in $\mathrm{eV}$, red - gross electron population.

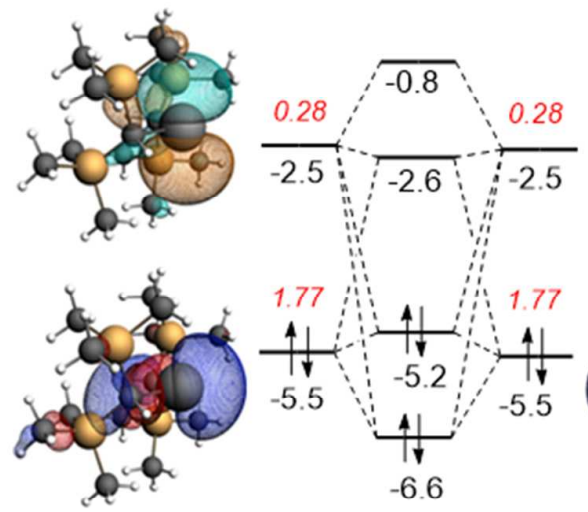

$\mathrm{Sn}(\mathrm{CH}(\mathrm{Si}(\mathrm{CH} 3) 3) 2) 2$ dimer $\Delta($ HOMO-LUMO $)=3.0$
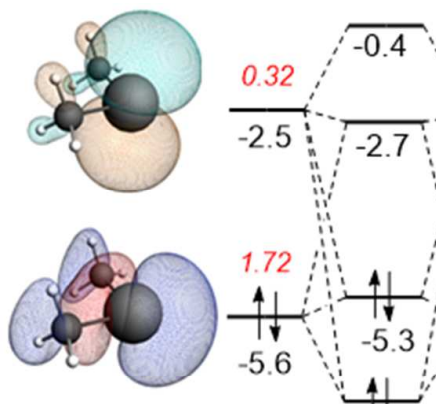

$-6.8$

$\mathrm{Sn}(\mathrm{CH} 3) 2$ dimer $\Delta(\mathrm{HOMO}-\mathrm{LUMO})=3.1$
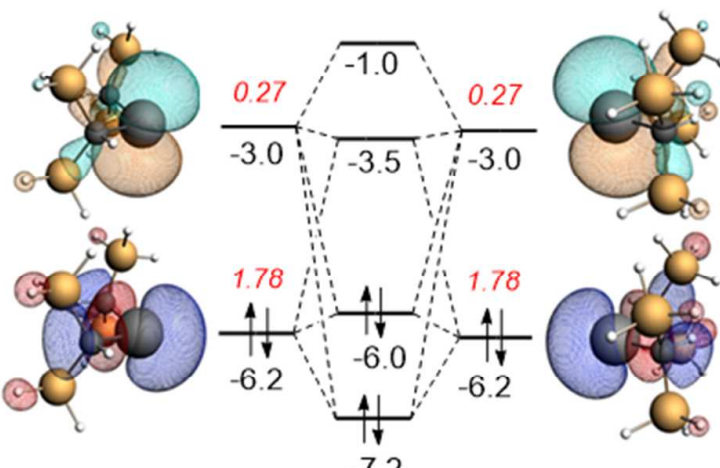

$\mathrm{Sn}(\mathrm{CH}(\mathrm{SiH} 3) 2) 2$ dimer $\triangle(\mathrm{HOMO}-\mathrm{LUMO})=3.2$

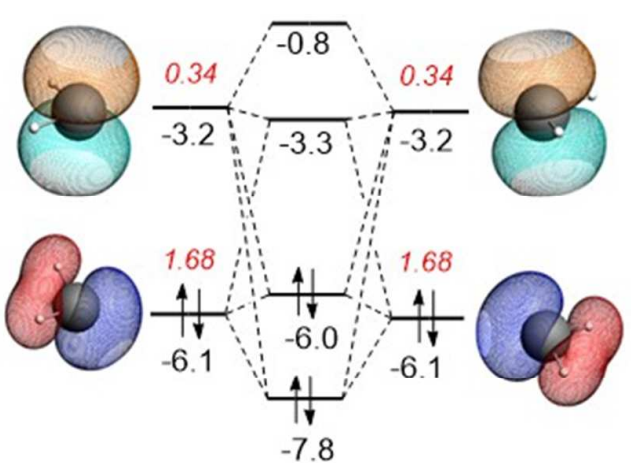

$\mathrm{SnH} 2$ dimer $\Delta$ (HOMO-LUMO $)=2.9$ 
Table S1. Data for MO analysis.

\begin{tabular}{lrrccc}
$N$ & Complex & $d(\mathrm{E}-\mathrm{E}) / \AA$ & $\Delta q$ & $\begin{array}{c}\Delta E(\mathrm{HOMO}- \\
\text { LUMO), eV }\end{array}$ & $\begin{array}{c}\text { Overlap } \\
<\text { HOMO/LUMO }>\end{array}$ \\
\hline $\mathrm{Sn}$ & & & & & \\
1b & $\mathrm{Sn}(\mathrm{CH}(\mathrm{Si}(\mathrm{CH} 3) 3) 2$ & 2.807 & 0.28 & 3.0 & 0.266 \\
2b & $\mathrm{SnCH}(\mathrm{SiH} 3) 2$ & 2.855 & 0.27 & 3.2 & 0.264 \\
3b & $\mathrm{Sn}(\mathrm{CH} 3) 2$ & 2.800 & 0.32 & 3.1 & 0.304 \\
4b & $\mathrm{SnH} 2$ & 2.748 & 0.34 & 2.9 & 0.314 \\
$\mathrm{Ge}$ & & & & & \\
1b & $\mathrm{Ge}(\mathrm{CH}(\mathrm{Si}(\mathrm{CH} 3) 3) 2$ & 2.366 & 0.39 & 3.1 & 0.270 \\
2b & $\mathrm{GeCH}(\mathrm{SiH} 3) 2$ & 2.393 & 0.36 & 3.2 & 0.287 \\
3b & $\mathrm{Ge}(\mathrm{CH} 3) 2$ & 2.344 & 0.36 & 3.2 & 0.314 \\
4b & $\mathrm{GeH} 2$ & 2.310 & 0.48 & 2.9 & 0.320 \\
\hline
\end{tabular}


xyz coordinates for all the complexes investigates

Sn: 1a:

114

$\begin{array}{llll}\text { Sn } & -0.23656 & 0.56357 & 1.24191\end{array}$

$\begin{array}{llll}\text { Si } & 2.56517 & 2.59904 & 1.98425\end{array}$

$\begin{array}{llll}\text { Si } & -0.39863 & 3.76248 & 2.25915\end{array}$

$\begin{array}{llll}\text { Si } & -1.54028 & -1.05577 & 3.85763\end{array}$

$\begin{array}{llll}\mathrm{Si} & 1.38136 & -2.02843 & 3.00466\end{array}$

$\begin{array}{llll}\text { C } & 2.87559 & 1.90819 & 3.67889\end{array}$

$\begin{array}{llll}\text { C } & 3.63701 & 1.73328 & 0.71949\end{array}$

$\begin{array}{llll}\text { C } & 3.18432 & 4.37267 & 2.06004\end{array}$

C $\quad 0.03666 \quad 5.50389 \quad 1.75460$

$\begin{array}{llll}\text { C } & -0.33652 & 3.60315 & 4.12801\end{array}$

$\begin{array}{llll}\text { C } & -2.17100 & 3.55095 & 1.72745\end{array}$

$\begin{array}{llll}\text { C } & -1.33448 & -1.48646 & 5.64278\end{array}$

$\begin{array}{llll}\text { C } & -2.74448 & 0.31826 & 3.75129\end{array}$

$\begin{array}{llll}\text { C } & -2.39037 & -2.48624 & 3.09403\end{array}$

$\begin{array}{llll}\text { C } & 0.70691 & -3.58183 & 2.24218\end{array}$

$\begin{array}{llll}\text { C } & 1.98578 & -2.40061 & 4.70496\end{array}$

$\begin{array}{llll}\text { C } & 2.90014 & -1.60711 & 1.97520\end{array}$

$\begin{array}{llll}\text { C } & 0.76143 & 2.53980 & 1.46500\end{array}$

C $\quad 0.11256 \quad-0.63693 \quad 3.06122$

$\begin{array}{llll}\mathrm{H} & 3.93901 & 1.95932 & 3.93682\end{array}$

$\mathrm{H} \quad 2.58155 \quad 0.86434 \quad 3.73319$

$\mathrm{H} \quad 2.32210 \quad 2.45907 \quad 4.43458$

$\mathrm{H} \quad 4.65177 \quad 1.74795 \quad 0.99552$

$\mathrm{H} \quad 3.49844 \quad 2.19026-0.26019$

$\begin{array}{llll}\mathrm{H} & 3.30190 & 0.68307 & 0.63351\end{array}$

H $\quad 4.18455 \quad 4.42104 \quad 2.36436$

$\mathrm{H} \quad 2.54423 \quad 4.92268 \quad 2.79424$

$\mathrm{H} \quad 3.03671 \quad 4.86001 \quad 1.09733$

$\begin{array}{llll}\mathrm{H} & -0.63164 & 6.22177 & 2.12679\end{array}$

$\mathrm{H} \quad-0.08470 \quad 5.55664 \quad 0.58826$

$\mathrm{H} \quad 1.05353 \quad 5.74652 \quad 1.93447$

$\mathrm{H} \quad-0.95756 \quad 4.32212 \quad 4.59296$

$\mathrm{H} \quad 0.71855 \quad 3.80524 \quad 4.44590$

$\begin{array}{llll}\mathrm{H} & -0.56927 & 2.60981 & 4.42327\end{array}$

$\begin{array}{llll}\mathrm{H} & -2.79413 & 4.27839 & 2.21729\end{array}$

$\begin{array}{llll}\mathrm{H} & -2.51010 & 2.55697 & 1.97972\end{array}$

$\mathrm{H} \quad-2.24857 \quad 3.68360 \quad 0.65614$

$\mathrm{H} \quad-2.23280 \quad-1.67517 \quad 6.12018$

H $\quad-0.85779 \quad-0.56444 \quad 6.15411$

$\begin{array}{llll}\mathrm{H} & -0.60510 & -2.26340 & 5.76948\end{array}$

$\mathrm{H} \quad-3.64493 \quad 0.10166 \quad 4.24227$

$\begin{array}{llll}\mathrm{H} & -2.87777 & 0.64967 & 2.74899\end{array}$

$\begin{array}{llll}\mathrm{H} & -2.28638 & 1.22245 & 4.31014\end{array}$

H $\quad-3.34578 \quad-2.69375 \quad 3.57482$

H $\quad-1.77360 \quad-3.39781 \quad 3.16756$

H $\quad-2.58882 \quad-2.31324 \quad 2.03629$ 


$\begin{array}{cccc}\mathrm{H} & 1.44380 & -4.35087 & 2.21729 \\ \mathrm{H} & 0.35996 & -3.38016 & 1.22177 \\ \mathrm{H} & -0.15879 & -3.93102 & 2.81686 \\ \mathrm{H} & 2.73257 & -3.23038 & 4.66084 \\ \mathrm{H} & 1.18295 & -2.73202 & 5.33960 \\ \mathrm{H} & 2.48631 & -1.54527 & 5.14728 \\ \mathrm{H} & 3.57234 & -2.45873 & 1.99104 \\ \mathrm{H} & 3.38850 & -0.74971 & 2.40961 \\ \mathrm{H} & 2.60139 & -1.39567 & 0.97289 \\ \mathrm{H} & 0.91835 & 2.87144 & 0.44120 \\ \mathrm{H} & 0.68404 & 0.00581 & 3.73319 \\ \mathrm{Sn} & 0.23656 & -0.56357 & -1.24191 \\ \mathrm{C} & -0.76143 & -2.53980 & -1.46500 \\ \mathrm{C} & -0.11256 & 0.63693 & -3.06122 \\ \mathrm{Si} & -2.56517 & -2.59904 & -1.98425 \\ \mathrm{Si} & 0.39863 & -3.76248 & -2.25915 \\ \mathrm{H} & -0.91835 & -2.87144 & -0.44120 \\ \mathrm{Si} & 1.54028 & 1.05577 & -3.85763 \\ \mathrm{Si} & -1.38136 & 2.02843 & -3.00466 \\ \mathrm{H} & -0.68404 & -0.00581 & -3.73319 \\ \mathrm{C} & -2.87559 & -1.90819 & -3.67889 \\ \mathrm{C} & -3.63701 & -1.73328 & -0.71949 \\ \mathrm{C} & -3.18432 & -4.37267 & -2.06004 \\ \mathrm{C} & -0.03666 & -5.50389 & -1.75460 \\ \mathrm{C} & 0.33652 & -3.60315 & -4.12801 \\ \mathrm{C} & 2.17100 & -3.55095 & -1.72745 \\ \mathrm{C} & 1.33448 & 1.48646 & -5.64278 \\ \mathrm{H} & 2.79413 & -4.27839 & -2.21729 \\ \mathrm{H} & 2.51010 & -2.55697 & -1.97972 \\ \mathrm{H} & 2.24857 & -3.68360 & -0.65614 \\ \mathrm{H} & 2.23280 & 1.67517 & -6.12018 \\ \mathrm{H} & 0.85779 & 0.56444 & -6.15411 \\ \mathrm{C} & 2.39037 & -0.31826 & -3.75129 \\ \mathrm{C} & -0.70691 & 3.58183 & -3.09403 \\ \mathrm{C} & -1.98578 & 2.40061 & -4.70496 \\ \mathrm{C} & -2.90014 & 1.60711 & -1.97520 \\ \mathrm{H} & -3.93901 & -1.95932 & -3.93682 \\ \mathrm{H} & -2.58155 & -0.86434 & -3.73319 \\ \mathrm{H} & -2.32210 & -2.45907 & -4.43458 \\ \mathrm{H} & -4.65177 & -1.74795 & -0.99552 \\ \mathrm{H} & -3.49844 & -2.19026 & 0.26019 \\ \mathrm{H} & -3.30190 & -0.68307 & -0.63351 \\ \mathrm{H} & -4.18455 & -4.42104 & -2.36436 \\ \mathrm{H} & -2.54423 & -4.92268 & -2.79424 \\ \mathrm{H} & -3.03671 & -4.86001 & -1.09733 \\ \mathrm{H} & 0.63164 & -6.22177 & -2.12679 \\ \mathrm{H} & 0.08470 & -5.55664 & -0.58826 \\ \mathrm{H} & 0.56953 & -5.74652 & -1.93447 \\ \mathrm{H} & -3.80524 & -4.59296 \\ \mathrm{H} & -2.44590 \\ \mathrm{H} & & \end{array}$




$\begin{array}{rrrr}\mathrm{H} & 0.60510 & 2.26340 & -5.76948 \\ \mathrm{H} & 3.64493 & -0.10166 & -4.24227 \\ \mathrm{H} & 2.87777 & -0.64967 & -2.74899 \\ \mathrm{H} & 2.28638 & -1.22245 & -4.31014 \\ \mathrm{H} & 3.34578 & 2.69375 & -3.57482 \\ \mathrm{H} & 1.77360 & 3.39781 & -3.16756 \\ \mathrm{H} & 2.58882 & 2.31324 & -2.03629 \\ \mathrm{H} & -1.44380 & 4.35087 & -2.21729 \\ \mathrm{H} & -0.35996 & 3.38016 & -1.22177 \\ \mathrm{H} & 0.15879 & 3.93102 & -2.81686 \\ \mathrm{H} & -2.73257 & 3.23038 & -4.66084 \\ \mathrm{H} & -1.18295 & 2.73202 & -5.33960 \\ \mathrm{H} & -2.48631 & 1.54527 & -5.14728 \\ \mathrm{H} & -3.57234 & 2.45873 & -1.99104 \\ \mathrm{H} & -3.38850 & 0.74971 & -2.40961 \\ \mathrm{H} & -2.60139 & 1.39567 & -0.97289\end{array}$

Sn: 1b:

114

$\begin{array}{lccc} & & & \\ \mathrm{Sn} & -0.016172 & -0.039892 & 0.055830 \\ \mathrm{Si} & -3.167469 & 1.107668 & -1.206187 \\ \mathrm{Si} & -2.973042 & -0.204515 & 1.724590 \\ \mathrm{Si} & 1.717880 & 2.439459 & 1.513165 \\ \mathrm{Si} & 1.298118 & 2.780342 & -1.643709 \\ \mathrm{C} & -2.673327 & 2.922259 & -0.962606 \\ \mathrm{C} & -2.853574 & 0.584827 & -2.998396 \\ \mathrm{C} & -5.049660 & 1.038957 & -0.944226 \\ \mathrm{C} & -4.617463 & -1.146158 & 1.729648 \\ \mathrm{C} & -3.204887 & 1.529712 & 2.447532 \\ \mathrm{C} & -1.800609 & -1.186221 & 2.851647 \\ \mathrm{C} & 2.415887 & 4.201835 & 1.546168 \\ \mathrm{C} & 0.673812 & 2.201779 & 3.078187 \\ \mathrm{C} & 3.154344 & 1.210834 & 1.552318 \\ \mathrm{C} & 3.146855 & 2.401078 & -1.820034 \\ \mathrm{C} & 1.031232 & 4.658754 & -1.702485 \\ \mathrm{C} & 0.433759 & 2.070792 & -3.169333 \\ \mathrm{C} & -2.275510 & -0.080560 & -0.035321 \\ \mathrm{C} & 0.624753 & 2.120516 & -0.000016 \\ \mathrm{H} & -3.413668 & 3.567604 & -1.454837 \\ \mathrm{H} & -1.696175 & 3.156114 & -1.394238 \\ \mathrm{H} & -2.648383 & 3.198545 & 0.098411 \\ \mathrm{H} & -3.176512 & 1.364900 & -3.700840 \\ \mathrm{H} & -3.417128 & -0.326994 & -3.230751 \\ \mathrm{H} & -1.795718 & 0.378759 & -3.191643 \\ \mathrm{H} & -5.548141 & 1.668971 & -1.694038 \\ \mathrm{H} & -5.340988 & 1.408544 & 0.046399 \\ \mathrm{H} & -5.441929 & 0.020117 & -1.049266 \\ \mathrm{H} & -4.955407 & -1.320544 & 2.760502 \\ \mathrm{H} & -4.497541 & -2.126960 & 1.250577\end{array}$




\begin{tabular}{|c|c|c|c|}
\hline $\mathrm{H}$ & -5.409704 & -0.608209 & 1.199827 \\
\hline $\mathrm{H}$ & -3.548506 & 1.479833 & 3.489107 \\
\hline $\mathrm{H}$ & -3.943074 & 2.108720 & 1.879142 \\
\hline $\mathrm{H}$ & -2.261532 & 2.089533 & 2.437422 \\
\hline $\mathrm{H}$ & -2.232993 & -1.292437 & 3.856108 \\
\hline $\mathrm{H}$ & -0.819110 & -0.707826 & 2.967270 \\
\hline 11 & -1.624404 & -2.193604 & 2.455224 \\
\hline $\mathrm{H}$ & 2.990453 & 4.361616 & 2.468877 \\
\hline $\mathrm{H}$ & 1.616760 & 4.953104 & 1.515516 \\
\hline $\mathrm{H}$ & 3.085415 & 4.393754 & 0.699587 \\
\hline $\mathrm{H}$ & 1.269084 & 2.381074 & 3.983532 \\
\hline $\mathrm{H}$ & 0.271142 & 1.183202 & 3.147550 \\
\hline $\mathrm{H}$ & -0.177734 & 2.894194 & 3.098274 \\
\hline $\mathrm{H}$ & 3.778485 & 1.360966 & 2.443226 \\
\hline $\mathrm{H}$ & 3.797373 & 1.312485 & 0.671158 \\
\hline $\mathrm{H}$ & 2.788838 & $0.1^{\prime}$ & 717 \\
\hline $\mathrm{H}$ & 3.509300 & 2.738029 & -2.800153 \\
\hline $\mathrm{H}$ & 3.342338 & 1.324991 & 9594 \\
\hline $\mathrm{H}$ & 3.749797 & 2.8 & -1.0 \\
\hline $\mathrm{H}$ & 1.392946 & 5.0 & -2. \\
\hline $\mathrm{H}$ & 1.552379 & 5.1 & -0.8 \\
\hline $\mathrm{H}$ & -0.036796 & 4.899909 & -1.622474 \\
\hline $\mathrm{H}$ & 0.877442 & 2.503451 & -4.076570 \\
\hline $\mathrm{H}$ & -0.635876 & 2.3 & -3.1 \\
\hline $\mathrm{H}$ & 0.5 & 0.9 & -3.2 \\
\hline $\mathrm{H}$ & -2.433331 & -1.078056 & -0.480360 \\
\hline $\mathrm{H}$ & -0.323669 & 2.646309 & 0.178833 \\
\hline $\mathrm{Sn}$ & 1.0 & -1.7 & -1.8 \\
\hline $\mathrm{C}$ & 3.3 & -1.7 & 4787 \\
\hline $\mathrm{C}$ & 0.41 & -3.9 & -1.829668 \\
\hline $\mathrm{Si}$ & 4.205747 & -2.908073 & -0.623776 \\
\hline $\mathrm{Si}$ & 4.011555 & -1.596103 & -3.5 \\
\hline $\mathrm{H}$ & 3.47 & -0.7 & 9837 \\
\hline $\mathrm{Si}$ & -0.680163 & -4.2 & -3.3 \\
\hline $\mathrm{Si}$ & -0.259666 & -4.580861 & -0.185800 \\
\hline $\mathrm{H}$ & 1.361716 & -4.447028 & -2.0 \\
\hline $\mathrm{C}$ & 3.71 & -4.7 & -0.86 \\
\hline $\mathrm{C}$ & 3.891812 & -2.3 & 1.168397 \\
\hline $\mathrm{C}$ & 6.087957 & -2.839335 & -0.885629 \\
\hline $\mathrm{C}$ & 5.655977 & -0.654448 & -3.559505 \\
\hline $\mathrm{C}$ & 4.24 & -3.330328 & -4.277507 \\
\hline $\mathrm{C}$ & 2.839216 & -0.614440 & -4.681813 \\
\hline $\mathrm{C}$ & -1.377892 & -6.002444 & -3.375801 \\
\hline $\mathrm{C}$ & 0.363293 & -4.001362 & -4.907892 \\
\hline $\mathrm{C}$ & -2.116860 & -3.011585 & -3.381100 \\
\hline $\mathrm{C}$ & -2.108393 & -4.201683 & -0.009197 \\
\hline $\mathrm{C}$ & 0.007265 & -6.459257 & -0.127060 \\
\hline $\mathrm{C}$ & 0.604908 & -3.871091 & 1.339586 \\
\hline $\mathrm{H}$ & 4.451860 & -5.367986 & -0.374825 \\
\hline $\mathrm{H}$ & 2.734386 & -4.956518 & -0.436062 \\
\hline $\mathrm{H}$ & 3.687118 & -4.999039 & -1.928372 \\
\hline $\mathrm{H}$ & 4.214043 & -3.165376 & 1.870917 \\
\hline
\end{tabular}




$\begin{array}{lrrr}\mathrm{H} & 4.455970 & -1.473665 & 1.400869 \\ \mathrm{H} & 2.834062 & -2.178282 & 1.361403 \\ \mathrm{H} & 6.586389 & -3.469319 & -0.135756 \\ \mathrm{H} & 6.379380 & -3.208952 & -1.876215 \\ \mathrm{H} & 6.480193 & -1.820485 & -0.780574 \\ \mathrm{H} & 5.993856 & -0.479784 & -4.590334 \\ \mathrm{H} & 5.536114 & 0.326220 & -3.080150 \\ \mathrm{H} & 6.448247 & -1.192566 & -3.029892 \\ \mathrm{H} & 4.587209 & -3.280392 & -5.319102 \\ \mathrm{H} & 4.981999 & -3.909160 & -3.709142 \\ \mathrm{H} & 3.300431 & -3.890369 & -4.267329 \\ \mathrm{H} & 3.271818 & -0.507895 & -5.686143 \\ \mathrm{H} & 1.857872 & -1.093070 & -4.797769 \\ \mathrm{H} & 2.662679 & 0.392809 & -4.285189 \\ \mathrm{H} & -1.953017 & -6.161989 & -4.298204 \\ \mathrm{H} & -0.578669 & -6.753641 & -3.345873 \\ \mathrm{H} & -2.046899 & -6.194699 & -2.528879 \\ \mathrm{H} & -0.231661 & -4.182798 & -5.813019 \\ \mathrm{H} & 0.763417 & -2.981820 & -4.978099 \\ \mathrm{H} & 1.216542 & -4.691673 & -4.927541 \\ \mathrm{H} & -2.740685 & -3.161017 & -4.272343 \\ \mathrm{H} & -2.760178 & -3.113979 & -2.500234 \\ \mathrm{H} & -1.751407 & -1.978298 & -3.403564 \\ \mathrm{H} & -2.470492 & -4.537775 & 0.971340 \\ \mathrm{H} & -2.304048 & -3.125704 & -0.080607 \\ \mathrm{H} & -2.711465 & -4.700650 & -0.776569 \\ \mathrm{H} & -0.354399 & -6.868693 & 0.826029 \\ \mathrm{H} & -0.513964 & -6.977833 & -0.938438 \\ \mathrm{H} & 1.075273 & -6.700459 & -0.207181 \\ \mathrm{H} & 0.161349 & -4.303437 & 2.247032 \\ \mathrm{H} & 1.674547 & -4.103819 & 1.350900 \\ \mathrm{H} & 0.501414 & -2.781685 & 1.404297\end{array}$

Sn: 2a:

42

$\begin{array}{lccc}\mathrm{H} & 0.002196 & -0.009747 & 0.355284 \\ \mathrm{Si} & 0.001654 & -0.003855 & 1.844190 \\ \mathrm{C} & 1.721439 & -0.001608 & 2.598471 \\ \mathrm{Si} & 2.690068 & 1.587861 & 2.516573 \\ \mathrm{H} & 4.064157 & 1.471497 & 3.079890 \\ \mathrm{H} & -0.792666 & -1.146096 & 2.367567 \\ \mathrm{H} & -0.749015 & 1.227118 & 2.225685 \\ \mathrm{H} & 2.037172 & 2.642618 & 3.339164 \\ \mathrm{H} & 2.813082 & 2.047325 & 1.107143 \\ \mathrm{Sn} & 3.062694 & -1.740692 & 2.240897 \\ \mathrm{C} & 3.138483 & -2.167700 & 0.076436 \\ \mathrm{Si} & 2.169859 & -3.636767 & -0.596568 \\ \mathrm{H} & 0.852999 & -3.826461 & 0.069755 \\ \mathrm{H} & 2.882751 & -4.930302 & -0.406146 \\ \mathrm{H} & 1.888420 & -3.441238 & -2.045192\end{array}$




$\begin{array}{lccc}\mathrm{Si} & 4.911814 & -1.997600 & -0.530147 \\ \mathrm{H} & 5.753279 & -3.196976 & -0.275804 \\ \mathrm{H} & 4.960434 & -1.772401 & -2.000674 \\ \mathrm{H} & 5.638504 & -0.874627 & 0.124726 \\ \mathrm{H} & 2.520581 & -1.402447 & -0.396675 \\ \mathrm{H} & 1.401956 & -0.124673 & 3.630731 \\ \mathrm{Sn} & 2.578548 & -3.807426 & 4.017936 \\ \mathrm{C} & 2.502771 & -3.380410 & 6.182396 \\ \mathrm{Si} & 3.471387 & -1.911332 & 6.855386 \\ \mathrm{H} & 4.788241 & -1.721629 & 6.189053 \\ \mathrm{C} & 3.919803 & -5.546510 & 3.660362 \\ \mathrm{Si} & 2.951167 & -7.135975 & 3.742257 \\ \mathrm{H} & 1.577085 & -7.019609 & 3.178926 \\ \mathrm{Si} & 5.639588 & -5.544263 & 4.414643 \\ \mathrm{H} & 6.390249 & -6.775248 & 4.033168 \\ \mathrm{Si} & 0.729446 & -3.550521 & 6.788991 \\ \mathrm{H} & -0.112031 & -2.351154 & 6.534650 \\ \mathrm{H} & 5.639046 & -5.538371 & 5.903548 \\ \mathrm{H} & 6.433916 & -4.402036 & 3.891247 \\ \mathrm{H} & 3.604067 & -8.190738 & 2.919678 \\ \mathrm{H} & 2.828136 & -7.595432 & 5.151688 \\ \mathrm{H} & 0.680838 & -3.775714 & 8.259520 \\ \mathrm{H} & 0.002761 & -4.673504 & 6.134129 \\ \mathrm{H} & 2.758483 & -0.617803 & 6.664964 \\ \mathrm{H} & 3.752838 & -2.106852 & 8.304009 \\ \mathrm{H} & 4.239272 & -5.423455 & 2.628096 \\ \mathrm{H} & 3.120682 & -4.145655 & 6.655505\end{array}$

Sn: 2b:

42

$\begin{array}{lrrr}\text { Sn } & 0.000000000 & 0.000000000 & 0.000000000 \\ \text { Si } & -2.911648296 & 0.389810638 & -1.723086177 \\ \text { Si } & -2.967401211 & 1.101663558 & 1.346628391 \\ \text { Si } & 1.215550782 & 2.770538075 & 1.599914653 \\ \text { Si } & 1.340772871 & 2.740838247 & -1.533931498 \\ \mathrm{H} & -2.305292217 & 1.647653898 & -2.232575646 \\ \mathrm{H} & -2.576585495 & -0.710465346 & -2.664751484 \\ \mathrm{H} & -4.391753275 & 0.569524815 & -1.705729606 \\ \mathrm{H} & -4.417703807 & 0.832662808 & 1.538607766 \\ \mathrm{H} & -2.805769191 & 2.531807380 & 0.971412551 \\ \mathrm{H} & -2.274895147 & 0.890561290 & 2.647171097 \\ \mathrm{H} & 1.164406946 & 4.249670731 & 1.750047735 \\ \mathrm{H} & 0.558366413 & 2.158362544 & 2.787582289 \\ \mathrm{H} & 2.639770637 & 2.342142211 & 1.593692594 \\ \mathrm{H} & 2.542668804 & 1.876335097 & -1.687242378 \\ \mathrm{H} & 1.812516073 & 4.152145173 & -1.444105808 \\ \mathrm{H} & 0.509694619 & 2.589647628 & -2.756415234 \\ \mathrm{C} & -2.244437116 & -0.000000000 & -0.000000000 \\ \mathrm{C} & 0.383045679 & 2.217482473 & 0.000000000 \\ \mathrm{H} & -2.510597439 & -1.044546858 & 0.224026074\end{array}$ 

$\mathrm{H} \quad-0.621585338 \quad 2.664726650 \quad-0.035079978$
Sn $\quad 1.013244547-1.139242057-2.413281566$
C $3.257696185-1.139025875-2.413290077$
$\begin{array}{llll}\text { C } & 0.630436212 & -3.356764080 & -2.413062840\end{array}$
Si $3.924942886-1.528741342-0.690197434$
$\begin{array}{lllll}\mathrm{Si} & 3.980755610 & -2.240637842 & -3.759906571\end{array}$
H $3.523739420-0.094451035-2.637330059$
Si $\quad-0.202013042 \quad-3.910035871 \quad-4.012936966$
Si $-0.327246996-3.880143427-0.879119732$
H $\quad 1.635117654 \quad-3.803889345 \quad-2.377947617$
H $\quad 3.318699112-2.786636426-0.180688172$
H $\quad 3.589765511 \quad-0.428468192 \quad 0.251457584$
H $\quad 5.405070503-1.708321583-0.707548106$
H $\quad 5.431024992-1.971483667 \quad-3.951929227$
H $3.819286498-3.670789091 \quad-3.384644157$
H $3.288193554-2.029649416-5.060439954$
$\mathrm{H} \quad-0.150622094 \quad-5.389166580-4.163010182$
H $\quad 0.455032365 \quad-3.297794563 \quad-5.200655632$
H $-1.626300033-3.481879351-4.006687177$
H $-1.529187938-3.015704911-0.725801265$
H $\quad-0.798922785-5.291468998-0.968924714$
H $\quad 0.503839955 \quad-3.728895444 \quad 0.343345708$

\section{Sn: 3a:}

18

$\begin{array}{llll}\text { C } & 0.083922 & 0.005476 & 0.030837\end{array}$

$\begin{array}{llll}\mathrm{Sn} & 0.020613 & -0.004064 & 2.255054\end{array}$

$\begin{array}{llll}\text { C } & 2.083279 & -0.021660 & 3.041231\end{array}$

$\begin{array}{llll}\mathrm{H} & 0.113268 & 1.031944 & -0.342478\end{array}$

$\mathrm{H} \quad 0.700996 \quad-0.737267 \quad-0.488442$

$\mathrm{H} \quad-0.893697 \quad-0.416705 \quad-0.265474$

$\begin{array}{llll}\mathrm{H} & 2.740728 & 0.016656 & 2.154449\end{array}$

$\begin{array}{llll}\mathrm{H} & 2.490549 & -0.958390 & 3.440407\end{array}$

$\begin{array}{llll}\mathrm{H} & 2.245722 & 0.870435 & 3.649555\end{array}$

$\begin{array}{llll}\text { Sn } & -1.822368 & -1.823536 & 3.233108\end{array}$

$\begin{array}{llll}\text { C } & -1.885678 & -1.833076 & 5.457325\end{array}$

$\begin{array}{llll}\text { C } & -3.885034 & -1.805925 & 2.446931\end{array}$

$\begin{array}{llll}\mathrm{H} & -4.292295 & -0.869194 & 2.047748\end{array}$

$\mathrm{H} \quad-4.542483 \quad-1.844229 \quad 3.333713$

$\begin{array}{llll}\mathrm{H} & -4.047486 & -2.698023 & 1.838613\end{array}$

$\mathrm{H} \quad-1.915024 \quad-2.859545 \quad 5.830640$

$\mathrm{H} \quad-0.908051 \quad-1.410913 \quad 5.753636$

$\mathrm{H} \quad-2.502751 \quad-1.090333 \quad 5.976604$

Sn: 3b:

18

$\begin{array}{llll}\text { C } & 0.072621 & 0.211601 & 0.068147\end{array}$ 


$\begin{array}{lrrc}\text { Sn } & -0.131011 & 0.159457 & 2.280550 \\ \mathrm{C} & 2.009324 & 0.029647 & 2.864589 \\ \mathrm{H} & 0.502321 & 1.176909 & -0.226080 \\ \mathrm{H} & 0.752047 & -0.586893 & -0.251966 \\ \mathrm{H} & -0.898148 & 0.087506 & -0.421645 \\ \mathrm{H} & 2.499516 & -0.751573 & 2.272047 \\ \mathrm{H} & 2.115805 & -0.195039 & 3.930349 \\ \mathrm{H} & 2.494697 & 0.989497 & 2.649821 \\ \mathrm{Sn} & -1.670913 & -1.987040 & 3.207359 \\ \mathrm{C} & -1.874082 & -2.039118 & 5.419808 \\ \mathrm{C} & -3.811376 & -1.857318 & 2.623773 \\ \mathrm{H} & -3.918099 & -1.632763 & 1.558010 \\ \mathrm{H} & -4.301448 & -1.076035 & 3.216331 \\ \mathrm{H} & -4.296680 & -2.817152 & 2.838770 \\ \mathrm{H} & -2.303600 & -3.004464 & 5.714177 \\ \mathrm{H} & -0.903225 & -1.914881 & 5.909390 \\ \mathrm{H} & -2.553537 & -1.240692 & 5.740025\end{array}$

Sn: 4a:

6

$\begin{array}{llll}\text { Sn } & -0.008549 & 0.041943 & 2.280693\end{array}$

$\begin{array}{llll}\mathrm{H} & 0.053469 & -0.135895 & 0.546129\end{array}$

$\begin{array}{llll}\mathrm{H} & 1.678347 & -0.087970 & 2.706959\end{array}$

$\begin{array}{llll}\text { Sn } & -1.564953 & -1.869571 & 3.493508\end{array}$

$\mathrm{H} \quad-1.626973 \quad-1.691734 \quad 5.228072$

H $\quad-3.251850 \quad-1.739663 \quad 3.067242$

Sn: 4b:

6

Sn $\quad 0.000000000 \quad 0.000000000 \quad 0.000000000$

$\mathrm{H} \quad-1.744654782 \quad 0.000000000-0.000000000$

H $\quad 0.350641463 \quad 1.709211271-0.000000000$

$\begin{array}{lllll}\text { Sn } & 1.064956447 & -1.303402691 & -2.171740823\end{array}$

H $2.809628423-1.303410828-2.171657245$

H $\quad 0.714315976 \quad-3.012563065 \quad-2.171774132$

Ge: 1a:

114

Ge $\quad 0.000000000 \quad 0.000000000 \quad 0.000000000$

C $-2.042614408 \quad 0.000000000-0.000000000$

C $\quad 0.757009564 \quad 1.828966227 \quad-0.000000000$

H $-3.184676790 \quad 3.198123582-2.116100581$

H $\quad-2.951610473 \quad 3.117402131 \quad-0.370049618$ 


\begin{tabular}{|c|c|c|c|}
\hline $\mathrm{H}$ & -1.574179822 & 2.906719135 & -1.446353 \\
\hline $\mathrm{H}$ & -3.003722561 & 0.691868894 & -3.798420114 \\
\hline $\mathrm{H}$ & -1.383884477 & 0.383492971 & -3.174026912 \\
\hline $\mathrm{H}$ & -2.623944882 & -0.863956810 & -3.044689962 \\
\hline $\mathrm{H}$ & -5.286218518 & 1.102381750 & -2.024569046 \\
\hline$\alpha$ & -4.985389918 & -0.452112352 & -1.237994059 \\
\hline $\mathrm{H}$ & -5.138267151 & 1.020351532 & -0.272088172 \\
\hline $\mathrm{H}$ & -4.898539384 & -0.165265822 & 2.895963806 \\
\hline 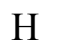 & -5.127322982 & 0.202975092 & 1.191022817 \\
\hline 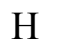 & -4.595620073 & -1.400020405 & 1.682696417 \\
\hline $\mathrm{H}$ & -3.046382702 & 2.153440955 & 3.218717165 \\
\hline $\mathrm{H}$ & -1.541956236 & 2.300731225 & 2.317306348 \\
\hline $\mathrm{H}$ & -3.079815219 & 2.615373171 & 1.514500533 \\
\hline 11 & -2.250792457 & -0.690014504 & 3.979881254 \\
\hline $\mathrm{H}$ & -1.848164005 & -1.845484173 & 2.711235987 \\
\hline $\mathrm{H}$ & -0.763773699 & -0.502434890 & 3.057912370 \\
\hline $\mathrm{H}$ & 2.297857770 & 4.215558528 & 2.827995859 \\
\hline $\mathrm{H}$ & 1.921176380 & 4.613726262 & 1.149716346 \\
\hline $\mathrm{H}$ & 0.628837049 & 4.222265659 & 2.2802 \\
\hline $\mathrm{H}$ & 1.901986636 & 1.417775330 & 3.852297218 \\
\hline $\mathrm{H}$ & 0.247434935 & 1.352869279 & 3.251519187 \\
\hline $\mathrm{H}$ & 1.406925132 & 0.124492895 & 2.749892603 \\
\hline $\mathrm{H}$ & 4.100935772 & 2.270627736 & 2.342967577 \\
\hline $\mathrm{H}$ & 3.812768829 & 0.882493809 & 5563 \\
\hline $\mathrm{H}$ & 4.036091976 & 2.489255019 & 0.594674902 \\
\hline $\mathrm{H}$ & 3.674374921 & 2.676950138 & -2.744487823 \\
\hline $\mathrm{H}$ & 3.871735481 & 2.592317645 & -0.999839651 \\
\hline $\mathrm{H}$ & 3.439240728 & $1.141 \mathrm{c}$ & -1.907 \\
\hline $\mathrm{H}$ & 1.844041075 & 4.952227227 & -2.282034010 \\
\hline $\mathrm{H}$ & 0.407945596 & 4.814232084 & -1.256848716 \\
\hline $\mathrm{H}$ & 2.020264171 & 4.773931214 & -0.542614557 \\
\hline $\mathrm{H}$ & 1.137086471 & 2.548502914 & -4.005262962 \\
\hline $\mathrm{H}$ & 0.794031089 & 0.984733302 & -3.252508645 \\
\hline $\mathrm{H}$ & -0.382739909 & 2.306213424 & -3.135027451 \\
\hline $\mathrm{H}$ & -1.981187188 & -1.076662298 & 0.047262012 \\
\hline $\mathrm{H}$ & -0.290078128 & 2.212301763 & 0.331974966 \\
\hline $\mathrm{Si}$ & -2.942502740 & 0.893333795 & -1.347302824 \\
\hline $\mathrm{Si}$ & -2.695137165 & 0.236273907 & 1.779800678 \\
\hline $\mathrm{Si}$ & 1.770616164 & 2.244095556 & 1.559056721 \\
\hline $\mathrm{Si}$ & 1.478485776 & 2.630162163 & -1.598263688 \\
\hline $\mathrm{C}$ & -2.669440977 & 2.730492951 & -1.283361105 \\
\hline $\mathrm{C}$ & -2.458473128 & 0.227302257 & -3.011140417 \\
\hline $\mathrm{C}$ & -4.822576042 & 0.649804502 & -1.209028591 \\
\hline $\mathrm{C}$ & -4.497956989 & -0.300046080 & 1.909215115 \\
\hline $\mathrm{C}$ & -2.607057652 & 2.025518333 & 2.292552704 \\
\hline $\mathrm{C}$ & -1.785726460 & -0.806646809 & 3.031769213 \\
\hline $\mathrm{C}$ & 1.610827975 & 4.021816341 & 1.959866719 \\
\hline $\mathrm{C}$ & 1.257471936 & 1.268977813 & 3.027833840 \\
\hline $\mathrm{C}$ & 3.588944575 & 1.885870590 & 1.402540223 \\
\hline $\mathrm{C}$ & 3.261167566 & 2.226991522 & -1.890632708 \\
\hline $\mathrm{C}$ & 1.391558425 & 4.435598784 & -1.474866579 \\
\hline $\mathrm{C}$ & 0.611068755 & 2.132119113 & -3.125985432 \\
\hline
\end{tabular}


Ge $\quad 0.944224712-1.745870200-1.250276924$

C $2.986839120-1.745870200-1.250276924$

C $\quad 0.187215148 \quad-3.574836426-1.250276924$

H $\quad 2.925411901-0.669207901-1.297538936$

Si $\quad 3.886727452 \quad-2.639203995 \quad 0.097025900$

Si $\quad 3.639361877-1.982144107-3.030077602$

H $\quad 1.234302841 \quad-3.958171963 \quad-1.582251890$

Si $\quad-0.826391451 \quad-3.989965756 \quad-2.809333645$

$\begin{array}{lllll}\text { Si } & -0.534261064 & -4.376032363 & 0.347986764\end{array}$

$\begin{array}{llll}\text { C } & 3.613665689 & -4.476363151 & 0.033084181\end{array}$

$\begin{array}{llll}\text { C } & 3.402697840 & -1.973172457 & 1.760863493\end{array}$

$\begin{array}{llll}\text { C } & 5.766800755 & -2.395674702 & -0.041248333\end{array}$

$\begin{array}{llll}\text { C } & 5.442181701 & -1.445824120 & -3.159492039\end{array}$

$\begin{array}{llll}\text { C } & 3.551282365 & -3.771388533 & -3.542829628\end{array}$

$\begin{array}{llll}\text { C } & 2.729951173 & -0.939223390 & -4.282046137\end{array}$

$\begin{array}{lllll}\text { C } & -0.666603263 & -5.767686540 & -3.210143643\end{array}$

C $\quad-0.313247223 \quad-3.014848013 \quad-4.278110764$

$\begin{array}{llll}\text { C } & -2.644719862 & -3.631740789 & -2.652817147\end{array}$

C $\quad-2.316942853 \quad-3.972861722 \quad 0.640355784$

$\begin{array}{llll}\text { C } & -0.447333712 & -6.181468984 & 0.224589655\end{array}$

C $\quad 0.333155957 \quad-3.877989313 \quad 1.875708508$

H $\quad 4.128901502 \quad-4.943993782 \quad 0.865823657$

H $3.895835186 \quad-4.863272331 \quad-0.880227306$

H $\quad 2.518404534 \quad-4.652589335 \quad 0.196076883$

H $\quad 3.947947273 \quad-2.437739093 \quad 2.548143190$

H $\quad 2.328109189-2.129363171 \quad 1.923749988$

H $3.568169594-0.881913390 \quad 1.794413038$

$\begin{array}{lllll}\mathrm{H} & 6.230443230 & -2.848251950 & 0.774292122\end{array}$

$\begin{array}{lllll}\mathrm{H} & 5.929614630 & -1.293757847 & -0.012282865\end{array}$

H $\quad 6.082491864 \quad-2.766221732-0.978188751$

H $\quad 5.842764096-1.580604378-4.146240730$

H $6.071547694-1.948845292 \quad-2.441299741$

H $5.539844786 \quad-0.345849795 \quad-2.932973341$

H $3.990607415 \quad-3.899311155-4.468994088$

H $2.486180948 \quad-4.046601425 \quad-3.567583272$

H $\quad 4.024039932-4.361243371 \quad-2.764777457$

H $3.195017169-1.055855696-5.230158178$

H $\quad 2.792388717 \quad 0.099613973 \quad-3.961512911$

H $\quad 1.707998411 \quad-1.243435310-4.308189294$

H $\quad-1.353633058 \quad-5.961428728 \quad-4.078272783$

H $\quad-0.976951668-6.359596462 \quad-2.399993269$

H $\quad 0.315387663 \quad-5.968135859 \quad-3.530521054$

$\mathrm{H} \quad-0.957761924-3.163645530 \quad-5.102574142$

H $\quad 0.696789777 \quad-3.098739479 \quad-4.501796111$

H $-0.462700420-1.870363095-4.000169527$

H $\quad-3.156711060 \quad-4.016497936-3.593244501$

H $\quad-2.868544117-2.628364009-2.533992487$

H $\quad-3.091867264-4.235125219-1.844951826$

H $\quad-2.730150209 \quad-4.422820338 \quad 1.494210899$

H $\quad-2.927510768 \quad-4.338187845 \quad-0.250437273$

H $\quad-2.495016015 \quad-2.887806438 \quad 0.657124227$

H $\quad-0.899816363-6.698097427 \quad 1.031757086$ 
H $\quad 0.536279116 \quad-6.560102284 \quad 0.006571792$

H $\quad-1.076039459-6.519801414-0.707662367$

H $\quad-0.192861758 \quad-4.294373114 \quad 2.754986038$

$\begin{array}{lllll}\mathrm{H} & 0.150193623 & -2.730603502 & 2.002231721\end{array}$

H $1.326964621 \quad-4.052083623 \quad 1.884750528$

\section{Ge: 1b:}

114

$\begin{array}{lrrr}\mathrm{H} & -2.245385542 & 4.294189986 & 1.471945902 \\ \mathrm{H} & -2.175658390 & 3.512652533 & -0.115890740 \\ \mathrm{H} & -2.483987100 & 2.550622012 & 1.335828922 \\ \mathrm{H} & -0.415950665 & 3.219770332 & 3.655971685 \\ \mathrm{H} & 0.348736121 & 1.694432655 & 3.186948448 \\ \mathrm{H} & 1.313523155 & 3.176653947 & 3.275978699 \\ \mathrm{H} & 0.348539904 & 5.584360419 & 1.703628856 \\ \mathrm{H} & 1.792756294 & 4.909347963 & 0.929211817 \\ \mathrm{H} & 0.386471866 & 5.360186259 & -0.052285125 \\ \mathrm{H} & 2.635047124 & 4.031457954 & -2.887337306 \\ \mathrm{H} & 2.049275288 & 4.811573762 & -1.406725780 \\ \mathrm{H} & 3.243711631 & 3.507655736 & -1.311164116 \\ \mathrm{H} & -0.522365041 & 3.505995201 & -3.532413610 \\ \mathrm{H} & -1.462881045 & 2.549403812 & -2.376168254 \\ \mathrm{H} & -0.924131045 & 4.177755273 & -1.943295341 \\ \mathrm{H} & 1.703307881 & 1.544637823 & -3.944998576 \\ \mathrm{H} & 2.544775188 & 0.767971921 & -2.593042238 \\ \mathrm{H} & 0.883306996 & 0.310427754 & -2.975278175 \\ \mathrm{H} & -4.833283770 & -0.835863624 & -2.812160191 \\ \mathrm{H} & -5.204636050 & -0.828794385 & -1.082986175 \\ \mathrm{H} & -4.617754159 & 0.647587953 & -1.864864645 \\ \mathrm{H} & -2.226875368 & -0.999774308 & -3.933896444 \\ \mathrm{H} & -1.528631557 & 0.458852984 & -3.209054659 \\ \mathrm{H} & -0.727743244 & -1.108865089 & -3.003293707 \\ \mathrm{H} & -2.697483603 & -3.355096173 & -2.197767167 \\ \mathrm{H} & -1.780177321 & -3.163441137 & -0.704175972 \\ \mathrm{H} & -3.547454284 & -3.197644229 & -0.652013644 \\ \mathrm{H} & -3.790025104 & -2.209920526 & 2.972753679 \\ \mathrm{H} & -4.187697433 & -2.468690099 & 1.268630145 \\ \mathrm{H} & -2.549092447 & -2.781907893 & 1.853713522 \\ \mathrm{H} & -5.142171575 & 0.579801038 & 2.530686313 \\ \mathrm{H} & -4.487301336 & 1.715936569 & 1.340345277 \\ \mathrm{H} & -5.302419992 & 0.243660677 & 0.798583838 \\ \mathrm{H} & -2.601725967 & 0.030629128 & 4.062864436 \\ \mathrm{H} & -1.045860754 & -0.075553348 & 3.218292198 \\ \mathrm{H} & -1.978910349 & 1.426030423 & 3.170788607 \\ \mathrm{H} & 1.740705255 & 1.750151076 & 0.380958602 \\ \mathrm{H} & -2.248309027 & 1.052869729 & -0.241863780 \\ \mathrm{Si} & 0.000000000 & 0.000000000 & 0.000000000 \\ & -0.044393449 & 3.191983484 & 1.184550815\end{array}$




\begin{tabular}{|c|c|c|c|}
\hline $\mathrm{Si}$ & 0.982157389 & 3419 & -1. \\
\hline $\mathrm{Si}$ & -2.720626551 & -0.970557733 & -1.491905003 \\
\hline $\mathrm{Si}$ & -2.981946764 & -0.289469790 & 1.637159176 \\
\hline $\mathrm{C}$ & -1.912136946 & 3.392007343 & 0.941002913 \\
\hline $\mathrm{C}$ & 0.335411828 & 2.770687929 & 2.993200991 \\
\hline $\mathrm{C}$ & 0.696728589 & 4.918491790 & 0.901603919 \\
\hline $\mathrm{C}$ & 2.347439269 & 3.854023606 & -1.841875511 \\
\hline $\mathrm{C}$ & -0.631919083 & 3.259958995 & -2.467940249 \\
\hline $\mathrm{C}$ & 1.576143271 & 1.154996508 & -2.925445777 \\
\hline $\mathrm{C}$ & -4.509460877 & -0.444175892 & -1.838205917 \\
\hline $\mathrm{C}$ & -1.703692259 & -0.611259729 & -3.049449550 \\
\hline $\mathrm{C}$ & -2.686023233 & -2.843995412 & -1.226196287 \\
\hline $\mathrm{C}$ & -3.406984148 & -2.110609275 & 1.948232218 \\
\hline $\mathrm{C}$ & -4.630469942 & 0.651459105 & 1.561070885 \\
\hline $\mathrm{C}$ & -2.058431399 & 0.335895298 & 3.158386009 \\
\hline $\mathrm{C}$ & 0.718426091 & 1.909574728 & 00000 \\
\hline $\mathrm{C}$ & -2.035656462 & 0.000000000 & 000000 \\
\hline $\mathrm{Ge}$ & 1.152971879 & -1.448102511 & 1.474010091 \\
\hline $\mathrm{C}$ & 0.371572877 & -3.330941682 & 1.443018813 \\
\hline $\mathrm{C}$ & 3.1860 & -1.508321570 & 1475 \\
\hline $\mathrm{H}$ & -0.6543 & -3.117819565 & 95745 \\
\hline $\mathrm{Si}$ & 1.043453639 & -4.581083830 & 0.173626938 \\
\hline $\mathrm{Si}$ & 0.141910562 & -4.009486363 & 3.209904637 \\
\hline $\mathrm{H}$ & 3.384 & -2.561951622 & 1.695262610 \\
\hline $\mathrm{Si}$ & 3.8889 & -0.505225834 & 5866 \\
\hline $\mathrm{Si}$ & 4.116456931 & -1.224071669 & 083142 \\
\hline $\mathrm{C}$ & 2.901629884 & -4.883937790 & 0.365372540 \\
\hline $\mathrm{C}$ & 0.627156404 & -4.004019565 & 751723 \\
\hline $\mathrm{C}$ & 0.220659488 & -6.281542260 & 74101 \\
\hline $\mathrm{C}$ & -1.252949954 & -5.291586862 & 3.271080302 \\
\hline $\mathrm{C}$ & 1.753922861 & -4.798166511 & 3.817822430 \\
\hline $\mathrm{C}$ & -0.377147003 & -2.641209847 & 4.408528001 \\
\hline $\mathrm{C}$ & 5.756279892 & -0.784537643 & 3.082545719 \\
\hline $\mathrm{C}$ & 3.066936394 & -1.021632842 & 839657 \\
\hline $\mathrm{C}$ & 3.569434203 & 1.343940353 & 2.687995289 \\
\hline $\mathrm{C}$ & 4.535064364 & 0.604710401 & -0.476593688 \\
\hline $\mathrm{C}$ & 5.749526064 & -2.191942485 & -0.145067944 \\
\hline $\mathrm{C}$ & 3.188638943 & -1.825867867 & -1.729041908 \\
\hline $\mathrm{H}$ & 3.200173724 & -5.722497847 & -0.278577709 \\
\hline $\mathrm{H}$ & 3.157020685 & -5.150992272 & 1.397110328 \\
\hline $\mathrm{H}$ & 3.507921281 & -4.022009531 & 0.082967150 \\
\hline $\mathrm{H}$ & 1.377995679 & -4.348920983 & -2.305094108 \\
\hline $\mathrm{H}$ & 0.576189702 & -2.912556632 & -1.649236216 \\
\hline $\mathrm{H}$ & -0.343557558 & -4.407365786 & -1.892554402 \\
\hline $\mathrm{H}$ & 0.499432764 & -6.917696640 & -0.470169501 \\
\hline $\mathrm{H}$ & -0.873263978 & -6.210056780 & 0.401930663 \\
\hline $\mathrm{H}$ & 0.542121265 & -6.794165786 & 1.295875084 \\
\hline $\mathrm{H}$ & -1.503924345 & -5.507132558 & 4.318819758 \\
\hline $\mathrm{H}$ & -0.990589902 & -6.235622629 & 2.785879545 \\
\hline $\mathrm{H}$ & -2.160815711 & -4.905968853 & 2.789951848 \\
\hline $\mathrm{H}$ & 1.681567042 & -5.058134385 & 4.882077513 \\
\hline $\mathrm{H}$ & 2.607710786 & -4.119857614 & 3.698031434 \\
\hline
\end{tabular}




$\begin{array}{rrrr}\mathrm{H} & 1.983762564 & -5.717462882 & 3.265456285 \\ \mathrm{H} & -0.490511868 & -3.053490069 & 5.420699014 \\ \mathrm{H} & -1.339887996 & -2.210340504 & 4.113726098 \\ \mathrm{H} & 0.345437233 & -1.821608105 & 4.461281022 \\ \mathrm{H} & 6.113090958 & -0.318462554 & 4.011076821 \\ \mathrm{H} & 6.313600530 & -0.338217993 & 2.251017294 \\ \mathrm{H} & 6.013083530 & -1.850549281 & 3.123109917 \\ \mathrm{H} & 3.639927835 & -0.623294672 & 5.381223936 \\ \mathrm{H} & 2.997808778 & -2.108514593 & 4.657153404 \\ \mathrm{H} & 2.053292985 & -0.609576740 & 4.601228769 \\ \mathrm{H} & 3.706136173 & 1.866690416 & 3.643801151 \\ \mathrm{H} & 2.539445369 & 1.520821137 & 2.367475361 \\ \mathrm{H} & 4.236547111 & 1.803788173 & 1.953218203 \\ \mathrm{H} & 4.932348621 & 0.734389642 & -1.491958447 \\ \mathrm{H} & 5.298177055 & 0.959842040 & 0.225034950 \\ \mathrm{H} & 3.662024908 & 1.257521988 & -0.378604992 \\ \mathrm{H} & 6.279566639 & -2.084459410 & -1.101467820 \\ \mathrm{H} & 5.574762594 & -3.263077814 & 0.015530300 \\ \mathrm{H} & 6.415519943 & -1.841439937 & 0.649648514 \\ \mathrm{H} & 3.735086832 & -1.510272824 & -2.628075968 \\ \mathrm{H} & 2.178027760 & -1.413229037 & -1.788706645 \\ \mathrm{H} & 3.107201894 & -2.915465401 & -1.754608006\end{array}$

\section{Ge: 2a:}

42

Ge $\quad \begin{array}{llll}1.377042 & 1.343050 & -0.698873\end{array}$

$\begin{array}{llll}\text { C } & 0.687960 & 3.198315 & -0.735431\end{array}$

$\begin{array}{lllll}\mathrm{Si} & 0.114847 & 4.037397 & -2.373836\end{array}$

$\begin{array}{llll}\mathrm{H} & -0.511532 & 3.098919 & -3.338309\end{array}$

$\begin{array}{llll}\text { C } & -0.003753 & 0.010524 & 0.001165\end{array}$

$\begin{array}{llll}\mathrm{Si} & -0.004450 & 0.014580 & 1.911475\end{array}$

$\begin{array}{llll}\mathrm{H} & 1.363117 & 0.011009 & 2.490406\end{array}$

$\begin{array}{llll}\mathrm{Si} & -1.649417 & -0.103719 & -0.836575\end{array}$

H $\quad-2.484316 \quad-1.192105 \quad-0.253622$

$\begin{array}{llll}\mathrm{Si} & 1.622271 & 4.390932 & 0.420042\end{array}$

H $\quad 2.751723 \quad 5.108606 \quad-0.229960$

$\begin{array}{lllll}\mathrm{H} & -2.448488 & 1.138398 & -0.684891\end{array}$

$\begin{array}{llll}\mathrm{H} & -1.483123 & -0.437763 & -2.274919\end{array}$

$\begin{array}{llll}\mathrm{H} & -0.653916 & -1.213616 & 2.442452\end{array}$

$\begin{array}{llll}\mathrm{H} & -0.758168 & 1.182715 & 2.443046\end{array}$

$\begin{array}{llll}\mathrm{H} & 0.695058 & 5.427957 & 0.943966\end{array}$

$\begin{array}{llll}\mathrm{H} & 2.257835 & 3.701650 & 1.574261\end{array}$

$\mathrm{H} \quad \begin{array}{llll}1.216211 & 4.696572 & -3.122357\end{array}$

$\mathrm{H} \quad-0.873276 \quad 5.109272 \quad-2.074182$

$\mathrm{H} \quad \begin{array}{llll}0.760473 & -0.744095 & -0.107077\end{array}$

$\mathrm{H} \quad-0.160470 \quad 2.847938 \quad-0.020625$

$\begin{array}{llll}\mathrm{Ge} & 2.743254 & 0.481151 & -2.399876\end{array}$

$\begin{array}{llll}\text { C } & 3.432337 & -1.374114 & -2.363333\end{array}$

$\begin{array}{lllll} & \mathrm{Si} & 4.005438 & -2.213214 & -0.724932\end{array}$

$\begin{array}{llll}\mathrm{H} & 4.631807 & -1.274746 & 0.239558\end{array}$ 


$\begin{array}{lrrr}\mathrm{C} & 4.124049 & 1.813676 & -3.099913 \\ \mathrm{Si} & 4.124746 & 1.809621 & -5.010224 \\ \mathrm{H} & 2.757179 & 1.813191 & -5.589154 \\ \mathrm{Si} & 5.769713 & 1.927919 & -2.262173 \\ \mathrm{H} & 6.604613 & 3.016306 & -2.845127 \\ \mathrm{Si} & 2.498038 & -2.566719 & -3.518827 \\ \mathrm{H} & 1.368581 & -3.284402 & -2.868843 \\ \mathrm{H} & 6.568784 & 0.685802 & -2.413858 \\ \mathrm{H} & 5.603420 & 2.261964 & -0.823830 \\ \mathrm{H} & 4.774212 & 3.037817 & -5.541200 \\ \mathrm{H} & 4.878464 & 0.641485 & -5.541794 \\ \mathrm{H} & 3.425256 & -3.603737 & -4.042755 \\ \mathrm{H} & 1.862483 & -1.877424 & -4.673043 \\ \mathrm{H} & 2.904067 & -2.872398 & 0.023572 \\ \mathrm{H} & 4.993564 & -3.285084 & -1.024589 \\ \mathrm{H} & 3.359823 & 2.568296 & -2.991672 \\ \mathrm{H} & 4.280774 & -1.023728 & -3.078127\end{array}$

\section{Ge: 2b:}

42

Ge $\quad 0.000000000 \quad 0.000000000 \quad 0.000000000$

Si $\quad-2.741787501 \quad 0.721722915-1.592047688$

Si $\quad-2.691112291 \quad 0.841102215 \quad 1.555091235$

Si $\quad 1.598840133 \quad 2.361654090 \quad 1.491529483$

$\begin{array}{lllll}\mathrm{Si} & 1.257958442 & 2.549699289 & -1.628737534\end{array}$

$\mathrm{H} \quad-2.155650911 \quad 2.060948032 \quad-1.848590596$

H $-2.445833100-0.169286160-2.741374986$

$\begin{array}{lllll}\mathrm{H} & -4.218464827 & 0.884468155 & -1.472919977\end{array}$

$\begin{array}{lllll}\mathrm{H} & -4.144375482 & 0.569364421 & 1.703644328\end{array}$

$\begin{array}{llll}\mathrm{H} & -2.492995707 & 2.311622488 & 1.457366721\end{array}$

$\mathrm{H} \quad-1.982553801 \quad 0.359521183 \quad 2.770542633$

$\begin{array}{llll}\mathrm{H} & 1.757408445 & 3.832627762 & 1.643927886\end{array}$

$\begin{array}{llll}\mathrm{H} & 1.005741406 & 1.814635127 & 2.740598356\end{array}$

H $\quad 2.940568244 \quad 1.752784238 \quad 1.301551895$

H $\quad 2.509245722 \quad 1.795434908 \quad-1.896639491$

H $\quad 1.611851848 \quad 3.995035339-1.550539025$

H $\quad 0.322716561 \quad 2.342111721 \quad-2.760959802$

C $\quad-2.018430535 \quad 0.000000000-0.000000000$

C $\quad 0.504260748 \quad 1.961187565 \quad 0.000000000$

H $\quad-2.309725396-1.059689229 \quad 0.049402945$

$\mathrm{H} \quad-0.448300003 \quad 2.497567295 \quad 0.130339491$

Ge $\quad 0.990518178-1.282272827-1.760607040$

C $3.008837563-1.285354300 \quad-1.767226329$

C $\quad 0.481919986-3.242488764-1.757309301$

Si $\quad 3.738404272-1.998590511-0.174134076$

$\begin{array}{lllll}\mathrm{Si} & 3.674809891 & -2.135917089 & -3.320090499\end{array}$

H $\quad 3.301204698 \quad-0.226289751-1.823443718$

$\begin{array}{lllll}\mathrm{Si} & -0.626877449 & -3.640089779 & -3.239016910\end{array}$

Si $\quad-0.258459596 \quad-3.830786569-0.122180793$ 
$\begin{array}{lrrr}\mathrm{H} & 1.432034591 & -3.780984217 & -1.896373500 \\ \mathrm{H} & 3.157416258 & -3.338632470 & 0.089410995 \\ \mathrm{H} & 3.443090882 & -1.103872911 & 0.972489687 \\ \mathrm{H} & 5.215261584 & -2.156954937 & -0.297010301 \\ \mathrm{H} & 5.126685042 & -1.862304198 & -3.478277943 \\ \mathrm{H} & 3.480087360 & -3.606096437 & -3.211312165 \\ \mathrm{H} & 2.958678837 & -1.664066832 & -4.534953527 \\ \mathrm{H} & -0.792744034 & -5.110553962 & -3.388636540 \\ \mathrm{H} & -0.042396035 & -3.096626808 & -4.493667353 \\ \mathrm{H} & -1.964500011 & -3.025754910 & -3.038008896 \\ \mathrm{H} & -1.508238547 & -3.077567484 & 0.155422168 \\ \mathrm{H} & -0.611575043 & -5.276500191 & -0.197279439 \\ \mathrm{H} & 0.685359920 & -3.622226210 & 1.002653066\end{array}$

Ge: 3a:

18

$\begin{array}{llll}\text { C } & 0.077885 & 0.009722 & 0.030867\end{array}$

$\begin{array}{llll}\text { Ge } & 0.017496 & -0.002792 & 2.072550\end{array}$

$\begin{array}{llll}\text { C } & 1.823241 & -0.019285 & 2.883223\end{array}$

$\begin{array}{llll}\mathrm{H} & 2.217757 & 0.299094 & 1.903905\end{array}$

$\begin{array}{llll}\mathrm{H} & 2.050408 & 0.872667 & 3.478923\end{array}$

H $\quad 2.257655 \quad-0.927029 \quad 3.298027$

$\mathrm{H} \quad \begin{array}{llll}0.612402 & -0.780870 & -0.486369\end{array}$

$\begin{array}{llll}\mathrm{H} & 0.231144 & 1.033987 & -0.333510\end{array}$

$\mathrm{H} \quad-1.022471 \quad 0.064708 \quad 0.061401$

Ge $\quad-1.763355 \quad-1.247490 \quad 2.956920$

$\begin{array}{llll}\text { C } & -3.569103 & -1.231010 & 2.146253\end{array}$

$\mathrm{H} \quad-3.963615 \quad-1.549376 \quad 3.125577$

$\mathrm{H} \quad-3.796270 \quad-2.122971 \quad 1.550566$

$\begin{array}{llll}\mathrm{H} & -4.003519 & -0.323271 & 1.731438\end{array}$

C $\quad-1.823743 \quad-1.260003 \quad 4.998603$

$\begin{array}{llll}\mathrm{H} & -0.723387 & -1.314990 & 4.968069\end{array}$

$\begin{array}{llll}\mathrm{H} & -2.358261 & -0.469410 & 5.515839\end{array}$

$\begin{array}{llll}\mathrm{H} & -1.977003 & -2.284271 & 5.362981\end{array}$

\section{Ge: 3b:}

18

$\begin{array}{lllll}\text { C } & 0.024300 & -0.109409 & -0.007814\end{array}$

$\begin{array}{llll}\mathrm{Ge} & -0.117219 & 0.096351 & 1.983708\end{array}$

$\begin{array}{llll}\text { C } & 1.763829 & 0.048644 & 2.683062\end{array}$

$\begin{array}{llll}\mathrm{H} & 2.262184 & 0.997072 & 2.446637\end{array}$

$\mathrm{H} \quad 1.774498 \quad-0.088961 \quad 3.769327$

$\mathrm{H} \quad 2.328403 \quad-0.764048 \quad 2.210585$

$\mathrm{H} \quad \begin{array}{llll}0.675703 & -0.955088 & -0.258696\end{array}$

$\mathrm{H} \quad 0.462146 \quad 0.801235 \quad-0.435328$

$\mathrm{H} \quad-0.960980 \quad-0.267880 \quad-0.458468$

Ge $\quad-1.628642 \quad-1.346635 \quad 3.045759$ 


$\begin{array}{llll}\mathrm{C} & -3.509693 & -1.298926 & 2.346414 \\ \mathrm{H} & -4.008071 & -2.247325 & 2.582909 \\ \mathrm{H} & -3.520366 & -1.161395 & 1.260140 \\ \mathrm{H} & -4.074242 & -0.486187 & 2.818839 \\ \mathrm{C} & -1.770156 & -1.140884 & 5.037284 \\ \mathrm{H} & -0.784875 & -0.982412 & 5.487936 \\ \mathrm{H} & -2.421561 & -0.295209 & 5.288174 \\ \mathrm{H} & -2.207997 & -2.051533 & 5.464795\end{array}$

Ge: 4a:

6

Ge $\quad-1.172222 \quad-0.000010 \quad-0.039985$

$\mathrm{H} \quad \begin{array}{llll}-2.009720 & 1.209675 & 0.412512\end{array}$

$\mathrm{H} \quad-1.807694 \quad-1.344655 \quad 0.371113$

Ge $\quad \begin{array}{llll}\text { G } & 1.172222 & 0.000010 & 0.039985\end{array}$

$\mathrm{H} \quad 2.009729-1.209670 \quad-0.412510$

$\mathrm{H} \quad \begin{array}{llll}1.807693 & 1.344657 & -0.371109\end{array}$

Ge: 4b:

6

Ge $\quad 0.000000000 \quad 0.000000000 \quad 0.000000000$

$\mathrm{H} \quad-1.549117381 \quad 0.000000000 \quad 0.000000000$

$\mathrm{H} \quad 0.460685074 \quad 1.478865521 \quad 0.000000000$

Ge $\quad 0.977913529-1.327889185-1.617113120$

H $\quad 2.527023167 \quad-1.327173248 \quad-1.617678660$

H $\quad 0.517875795 \quad-2.807031382-1.616494891$ 